\title{
Dynamics of Carroll strings
}

\author{
Biel Cardona, ${ }^{a}$ Joaquim Gomis ${ }^{a, b}$ and Josep M. Pons ${ }^{a}$ \\ ${ }^{a}$ Departament d'Estructura $i$ Constituents de la Matèria \\ and Institut de Ciències del Cosmos (ICCUB) Facultat de Física, Universitat de Barcelona, \\ Diagonal 647, E-08028 Barcelona, Catalonia, Spain \\ ${ }^{b}$ Department of Physics, Faculty of Science, Chulalongkorn University, \\ Bangkok 10330, Thailand \\ E-mail: cardonarotger@gmail.com, gomis@ecm.ub.edu, pons@ecm.ub.edu
}

\begin{abstract}
We construct the canonical action of a Carroll string doing the Carroll limit of a canonical relativistic string. We also study the Killing symmetries of the Carroll string, which close under an infinite dimensional algebra. The tensionless limit and the Carroll $p$-brane action are also discussed.
\end{abstract}

KEYwords: Bosonic Strings, Space-Time Symmetries, p-branes

ARXIV EPRINT: 1605.05483 


\section{Contents}

1 Introduction 1

2 Canonical Carroll string action $\quad 2$

2.1 Carroll symmetries, Carroll string algebra and Carroll diffeomorphism 3

2.2 Dynamics 4

3 Killing symmetries of the Carroll string 5

4 Tensionless Carroll string $\quad 6$

5 Carroll $p$-brane action $\quad 7$

$\begin{array}{llr}6 & \text { Discussion and outlook } & 8\end{array}$

\section{Introduction}

The extension of the holographic ideas to non-AdS situations with applications to condensed matter systems has produced a renewed interest in the study non-relativistic symmetries $^{1}$ and the use of non-relativistic gravity theories in the bulk [6] like Newton-Cartan [7] and Horava gravities [8]. Recently these theories have been constructed from the gauging of the Bargmann algebra [9], from Lihshitz holography [10] and from the use non-relativistic conformal methods [11].

The study of space-time holography [12-15] has lead to reconsider the role of the BMS group [16-18]. On the other hand it has been shown that BMS symmetry is an infinite conformal extension of the Carroll symmetry [19, 20]. Carroll symmetry was introduced in [21-23] as the limit of the Poincaré algebra when the velocity of light tends to zero. There is a duality between the non-relativistic symmetry and the Carroll symmetry [24].

The strong coupling limit of gravity [25] introduced many years ago was the first example of a dynamical system possessing Carroll symmetry. More recently it has been constructed the action of the Carroll particle [24, 26, 27] and the Carroll superparticle [28], both exhibiting a trivial dynamics. Notice that in these cases the massless limit can be taken at the level of the action.

The reason for the trivial dynamics of the free particle Carroll objects is due to the fact that the light cone in the Carroll case collapses to the time vertical axis. Carroll symmetries appear also in warped conformal field theories [32]. The construction of a Carroll gravity by a modified gauging of the Carroll algebra has been studied recently in [33].

\footnotetext{
${ }^{1}$ The use of non-relativistic extended objects has been also studied as a soluble sector of string theory $[1-5]$.
} 
In this note we continue the study of dynamical objects with Carroll symmetry. We construct the action of a tension-full and a tensionless ${ }^{2}$ Carroll string by taking the Carrollian limits ${ }^{3}$ of the canonical action of a relativistic string. We will also construct the action of a Carroll $p$-brane by the same procedure. The action for these objects can be also constructed using the method of non-linear realizations [35, 36] applied to the Carroll algebras; for the Carroll algebra see [37], for string Carroll algebras [26]. In either of the two limits, the Carroll string exhibits a trivial dynamics like the Carroll particle. This result also applies to branes. We will also study the Killing symmetries of the Carroll string, and show that these symmetries close under an infinite dimensional algebra.

The organization of the paper is as follows, in section 2 we construct canonical Carroll string actions (at least two types of limits form the relativistic string are available). In section 3 we study their Killing symmetries. The tensionless limit is analyzed in section 4 and section 5 is devoted to construct a Carroll $p$-brane action. Finally we write some conclusions and outlook.

\section{Canonical Carroll string action}

Our starting point is the canonical action of a relativistic string:

$$
S_{\mathrm{NG}}=\int \mathrm{d}^{2} \sigma\left(p \cdot \dot{x}-\frac{\tilde{e}}{2} \tilde{H}-\tilde{\mu} \tilde{T}\right)=\int \mathrm{d} \tau \mathrm{d} \sigma\left(p \cdot \dot{x}-\frac{\tilde{e}}{2}\left(p^{2}+T_{r}^{2}{x^{\prime}}^{2}\right)-\tilde{\mu}\left(p \cdot x^{\prime}\right)\right),
$$

where $\tilde{H}=p^{2}+T_{r}^{2} x^{\prime 2}$ and $\tilde{T}=p \cdot x^{\prime}$ are the diffeomorphism constraints and $\tilde{e}, \tilde{\mu}$ are Lagrange multipliers. In order to obtain the Carroll action for the string we take the 'stringy' Carrollian limit by rescaling the longitudinal coordinates $x^{\mu}(\mu=0,1)$ with a dimensionless parameter $\omega$ :

$$
x^{\mu}=\frac{X^{\mu}}{\omega}, \quad p_{\mu}=\omega P_{\mu} .
$$

The action is obtained by plugging these expressions in (2.1) and taking the limit $\omega \rightarrow \infty$. Then the products $p \cdot \dot{x}$ and $p \cdot x^{\prime}$ remain unaffected and become $P \cdot \dot{X}$ and $P \cdot X^{\prime}$ respectively. All physics in the ultra-relativistic Carrollian regime arises from the constraint proportional to $e$, like in the Carroll particle [27]. We must rescale the einbein field as in the case of the Carroll particle, $\tilde{e}=e / \omega^{2}$, whereas $\tilde{\mu}=\mu$ remains the same. Rescaling the string tension as $T_{r}=\omega T$ and sending $\omega$ to infinity, we obtain the action of the Carroll string

$$
S_{\mathrm{C}_{s}}=\int \mathrm{d} \tau \mathrm{d} \sigma\left(P \cdot \dot{X}-\mu\left(P \cdot X^{\prime}\right)-\frac{e}{2}\left(\eta_{\mu \nu} P^{\mu} P^{\nu}+T^{2} X_{i}^{\prime 2}\right)\right) .
$$

where $X_{i}^{\prime 2}=\delta_{i j} X^{i^{\prime}} X^{j^{\prime}}, i, j=2, \ldots, D-1$. The transversality constraint does not change, whereas the mass-shell constraint becomes $\left(E=P^{0}\right)$ :

$$
\hat{H}=-E^{2}+\left(P^{1}\right)^{2}+T^{2} X_{i}^{\prime 2} .
$$

Notice the absence of the transverse momenta $P_{i}$.

\footnotetext{
${ }^{2}$ The situation regarding the tensionless limit is rather different here than in the case of the nonrelativistic limit [34].

${ }^{3}$ Like in the non-relativistic case, where there is not a unique limit for extended objects $[1,3,38]$, the same is true for the Carroll limit of an extended object.
} 
If instead we perform on the action (2.1) the Carroll limit à la particle [27], $x^{0}=t / \omega$, $p_{0}=-\omega E$, we get

$$
\tilde{S}_{\mathrm{C}_{s}}=\int \mathrm{d} \tau \mathrm{d} \sigma\left(P \cdot \dot{X}-\mu\left(P \cdot X^{\prime}\right)-\frac{e}{2}\left(-E^{2}+T^{2} X_{\hat{\imath}}^{\prime 2}\right)\right),
$$

$\hat{\imath}=1, \ldots, D-1$, thus the mass-shell constraint is now given by $\tilde{H}=-E^{2}+T^{2} X_{\hat{\imath}}^{\prime 2}$.

A physical difference between the two Carroll strings appears when we dimensionally reduce on $S^{1}$ : in one case we obtain a Carroll massless particle whereas in the other a massive Carroll particle [19, 20, 27]. Let us mention that in the non-relativistic case, the limits $\grave{a}$ la string or $\grave{a}$ la particle lead also to two different particle Lagrangians: the vibrating string (('stringy' NR limit) $[1,3,4]$ leads to the massive non-relativistic particle Lagrangian whereas the non-vibrating string with a fixed length (à la particle limit) [38, 39] leads to a particle Lagrangian which is just a total derivative.

\subsection{Carroll symmetries, Carroll string algebra and Carroll diffeomorphism}

The canonical action (2.3) is invariant under the 'stringy' Carroll transformations:

$$
\begin{aligned}
\delta X^{\mu} & =\omega^{\mu}{ }_{\nu} X^{\nu}+\omega^{\mu}{ }_{i} X^{i}+\zeta^{\mu}, & \delta X^{i} & =\omega^{i}{ }_{j} X^{j}+\zeta^{i}, \\
\delta P_{\mu} & =\omega_{\mu}{ }_{\mu} P_{\nu}, & \delta P_{i} & =\omega_{i}{ }^{\mu} P_{\mu}+\omega_{i}{ }^{j} P_{j},
\end{aligned}
$$

where $\left(\omega^{\mu}{ }_{\nu}, \omega^{\mu}{ }_{i}, \omega^{i}{ }_{j}, \zeta^{\mu}, \zeta^{i}\right)$ are respectively the Lorentz boosts in the two longitudinal directions, the time and space Carroll boosts, the spatial rotations, longitudinal translations and the transverse translations. These transformations can all be derived from a general infinitesimal Poincaré transformation, $\delta x^{M}=\omega_{N}^{M} x^{N}+\xi^{M}$ and $\delta p_{M}=\omega_{M}^{N} p_{N}$, by performing the rescaling

$$
x^{\mu}=\frac{X^{\mu}}{\lambda}, \quad \omega_{i}^{\mu} \rightarrow \frac{\omega^{\mu}}{\lambda}, \quad \xi^{\mu}=\frac{\zeta^{\mu}}{\lambda}, \quad p_{\mu}=\lambda P_{\mu},
$$

and taking the limit $\lambda \rightarrow \infty$.

The algebra of these transformations closes under what we call the String Carroll algebra

$$
\begin{aligned}
{\left[M_{i j}, P_{k}\right] } & =2 \delta_{k[j} P_{i]}, & {\left[M_{i j}, M_{k l}\right] } & =2 \delta_{i[k} M_{l] j}-2 \delta_{j[k} M_{l] i}, \\
{\left[K, P_{0}\right] } & =P_{1}, & {\left[K, P_{1}\right] } & =P_{0}, \\
{\left[K, K_{i}\right] } & =B_{i}, & {\left[K, B_{i}\right] } & =K_{i}, \\
{\left[M_{i j}, K_{k}\right] } & =2 \delta_{k[j} K_{i]}, & {\left[M_{i j}, B_{k}\right] } & =2 \delta_{k[j} B_{i]}, \\
{\left[K_{i}, P_{j}\right] } & =-\delta_{i j} P_{0}, & {\left[B_{i}, P_{j}\right] } & =-\delta_{i j} P_{1}
\end{aligned}
$$

where Lie algebra generators are the longitudinal Lorentz boost $K$, the time Carroll boosts $K_{i}$, the space Carroll boosts $B_{i}$, the spatial rotations $M_{i j}$, and the time-space translations $P_{0}, P_{1}, P_{i}$. This algebra can be obtained from the Poincaré algebra

$$
\begin{aligned}
{\left[M_{A B}, M_{C D}\right] } & =2 \eta_{A[C} M_{D] B}-2 \eta_{B[C} M_{D] A}, \\
{\left[M_{A B}, P_{C}\right] } & =2 \eta_{C[B} P_{A]}, \quad\left[P_{A}, P_{B}\right]=0,
\end{aligned}
$$


by the contraction

$$
P_{A} \rightarrow\left(\omega P_{0}, \omega P_{1}, P_{i}\right), \quad K_{i} \rightarrow \omega K_{i}, \quad B_{i} \rightarrow \omega B_{i}
$$

with the identifications $M_{01} \equiv K, M_{i 0} \equiv K_{i}, M_{i 1} \equiv B_{i}$.

The action has also the gauge invariance of diffeomorphisms. These are generated by the first class constraints $\hat{H}(\sigma)=-E^{2}+\left(P^{1}\right)^{2}+T^{2} X_{i}^{\prime 2}, \hat{T}(\sigma)=\left(P \cdot X^{\prime}\right)$, whose algebra is

$$
\begin{aligned}
\left\{\hat{H}(\sigma), \hat{H}\left(\sigma^{\prime}\right)\right\} & =0, \\
\left\{\hat{H}(\sigma), \hat{T}\left(\sigma^{\prime}\right)\right\} & =\hat{H}(\sigma) \partial_{\sigma} \delta\left(\sigma-\sigma^{\prime}\right)-\hat{H}\left(\sigma^{\prime}\right) \partial_{\sigma^{\prime}} \delta\left(\sigma-\sigma^{\prime}\right), \\
\left\{\hat{T}(\sigma), \hat{T}\left(\sigma^{\prime}\right)\right\} & =\hat{T}(\sigma) \partial_{\sigma} \delta\left(\sigma-\sigma^{\prime}\right)-\hat{T}\left(\sigma^{\prime}\right) \partial_{\sigma^{\prime}} \delta\left(\sigma-\sigma^{\prime}\right) .
\end{aligned}
$$

\subsection{Dynamics}

The action of the Carroll string is given by (2.3). The canonical Hamiltonian is:

$$
H_{D}=\int \mathrm{d} \sigma\left(\mu\left(\eta_{\mu \nu} P^{\mu} X^{\nu \prime}+P_{i} X^{i^{\prime}}\right)+\frac{e}{2}\left(\eta_{\mu \nu} P^{\mu} P^{\nu}+T^{2} X_{i}^{\prime 2}\right)\right) .
$$

The non-vanishing Poisson brackets are given by

$$
\begin{aligned}
& \left\{X^{M}(\sigma), P^{N}\left(\sigma^{\prime}\right)\right\}=\eta^{M N} \delta\left(\sigma-\sigma^{\prime}\right), \\
& \left\{X^{M}(\sigma), X^{N}\left(\sigma^{\prime}\right)\right\}=\left\{P_{M}(\sigma), P_{N}\left(\sigma^{\prime}\right)\right\}=0,
\end{aligned}
$$

the equations of motion follow:

$$
\begin{aligned}
\dot{X}^{\mu} & =\mu X^{\mu \prime}+e P^{\mu}, & \dot{P}^{\mu} & =\left(\mu P^{\mu}\right)^{\prime}, \\
\dot{X}^{i} & =\mu X^{i^{\prime}}, & \dot{P}^{i} & =\left(\mu P^{i}+e T^{2} X^{i^{\prime}}\right)^{\prime},
\end{aligned}
$$

and the constraints

$$
\eta_{\mu \nu} P^{\mu} X^{\nu \prime}+P_{i} X^{i^{\prime}}=0, \quad \eta_{\mu \nu} P^{\mu} P^{\nu}+T^{2} X_{i}^{\prime 2}=0 .
$$

As we can see the dynamics of the Carroll string is trivial. In fact considering the analogous of the conformal gauge, $e=1, \mu=0$, we have

$$
\begin{aligned}
\dot{X}^{\mu} & =P^{\mu}, \quad \dot{P}^{\mu}=0, \\
\dot{X}^{i} & =0, \quad \dot{P}^{i}=T^{2}\left(X^{i^{\prime}}\right)^{\prime} .
\end{aligned}
$$

We see that the transverse coordinates of the bosonic string are constant, therefore the free Carroll string does not move. Notice however that the momenta are not constant. This is a common feature of (free) Carroll particle, Carroll string or in general, Carroll $p$-brane (see section 5): in Carroll space there is no connection between spatial momenta and velocities.

If we consider Carroll strings coupled to Carroll gravity the strings will have a nontrivial dynamics like in the case of the Carroll particle coupled to Carroll gauge fields [27]. 


\section{Killing symmetries of the Carroll string}

In this section we analyze the Killing symmetries of the Carroll string. As we will see the string action is invariant under an infinite dimensional group of transformations that includes the String Carroll transformations (2.6). It turns out that the full symmetry group includes conformal symmetries in both the transverse and the longitudinal fields. In contrast to higher dimensions, the conformal algebra in two dimensions is infinite dimensional, hence longitudinal fields will have a infinite-dimensional symmetry, a common feature in the Carrollian context $[27,28]$. This is not the case for the transversal $D-2$ coordinates.

Let us consider the generator of canonical symmetry transformations

$$
G=\int \mathrm{d} \sigma\left(\xi^{M} P_{M}+\Lambda \pi_{e}+\gamma \pi_{\mu}\right),
$$

with $\xi^{M}, \Lambda$ and $\gamma$ arbitrary functions on the extended configuration space, which includes $e$ and $\mu$ as new variables. The extended phase space includes the momenta $\pi_{e}, \pi_{\mu}$, which are the new primary constraints. Conservation of $G$ reads:

$$
\begin{aligned}
0=\dot{G}= & \int \mathrm{d} \sigma\left(e P^{\mu} P^{\nu}\left(2 \partial_{(\mu} \xi_{\nu)}-\frac{\Lambda}{2 e} \eta_{\mu \nu}\right)+e\left(P^{i} P^{\mu}-T^{2} X^{\mu^{\prime}} X^{i^{\prime}}\right) \partial_{\mu} \xi_{i}\right. \\
& \left.-e T^{2} X^{i^{\prime}} X^{j^{\prime}}\left(2 \partial_{(i} \xi_{j)}+\frac{\Lambda}{2 e} \delta_{i j}\right)-\gamma\left(\eta_{\mu \nu} P^{\mu} X^{\nu \prime}+P_{i} X^{i^{\prime}}\right)\right) .
\end{aligned}
$$

The Killing equations are:

$$
\partial_{\mu} \xi_{\nu}+\partial_{\nu} \xi_{\mu}=\tilde{\lambda} \eta_{\mu \nu}, \quad \partial_{\mu} \xi_{i}=0, \quad \partial_{i} \xi_{j}+\partial_{j} \xi_{i}=-\tilde{\lambda} \delta_{i j}, \quad \gamma=0
$$

with $\Lambda$ determined as $\Lambda=e \tilde{\lambda}$ and with the conformal factor $\tilde{\lambda}$ (notice that $\tilde{\lambda}=\tilde{\lambda}\left(X^{i}\right)$ ) satisfying:

$$
\tilde{\lambda}=\partial_{\mu} \xi^{\mu}=-\frac{2}{D-2} \partial_{i} \xi^{i} .
$$

The second Killing equation tells us that $\xi^{i}=\xi^{i}\left(X^{j}\right)$. Notice also the sign difference in (3.3) between the conformal Killing equation for longitudinal vectors and transversal ones. In particular when we consider a scale transformation we have Lifshitz scaling with $z=-1: \delta X^{\mu}=-c X^{\mu}$ and $\delta X^{i}=c X^{i}$, where $c$ is the infinitesimal dilatation parameter. The equation for transverse fields is the Euclidean Conformal Killing equation. For $D=3$ there is only one transversal direction and hence no restriction on $\xi^{i}$. For $D=4$ we have the standard two-dimensional infinite conformal symmetry for the (two) transverse variables. For $D>4$, we get

$$
\tilde{\lambda}\left(X^{i}\right)=2\left(2 b_{k} X^{k}-c\right),
$$

for some constants $b_{k}$ and $c$. In this case the solution is:

$$
\xi^{i}\left(X^{k}\right)=a^{i}+\omega^{i}{ }_{j} X^{j}+c X^{i}+X_{j}^{2} b^{i}-2\left(b_{j} X^{j}\right) X^{i},
$$

where $a^{i}, \omega^{i}{ }_{j}, c$ and $b^{i}$ generate space translations, rotations, space dilatations and special conformal transformations, respectively. Notice as a feature of the rescaling $\omega \rightarrow \infty$ of 
the relativistic string to obtain the Carroll string (as done in section 2) that if we dimensionally reduce the Carroll string to the Carroll particle, this reduction at the level of the Killing symmetries does not reproduce the infinite-dimensional symmetry for transverse fields which exists in the particle case [27].

The solution to the first equation is:

$$
\xi^{\mu}(X)=\frac{\tilde{\lambda}}{2} X^{\mu}+\Omega^{\mu}{ }_{\nu}\left(X^{i}\right) X^{\nu}+f^{\mu}\left(X^{i}\right),
$$

where the antisymmetric tensor $\Omega_{\mu \nu}$ and the vector $f^{\mu}$ have arbitrary dependences on the transverse coordinates $X^{i}$.

In case we consider the action (2.5) for the Carroll string à la particle, the Killing equations give the following transformations

$$
\xi^{0}(X)=\frac{\tilde{\lambda}}{2} X^{0}+f^{0}\left(X^{i}\right)
$$

and the same results as before for the spatial components $\xi^{i}(X)$, but now for $i=$ $1,2, \ldots, D-1$.

\section{Tensionless Carroll string}

Like in the particle case where the massless limit can be taken straightforwardly, here we analyze the tensionless limit, ${ }^{4} T \rightarrow 0$, of the Carroll string action (2.3). In this limit the mass-shell becomes $\hat{H}_{0}=-\left(P^{0}\right)^{2}+\left(P^{1}\right)^{2}$ and the Dirac's Hamiltonian is:

$$
H_{D}(T \rightarrow 0)=\int \mathrm{d} \sigma\left(\mu\left(\eta_{\mu \nu} P^{\mu} X^{\nu \prime}+P_{i} X^{i^{\prime}}\right)+\frac{e}{2} \eta_{\mu \nu} P^{\mu} P^{\nu}\right) .
$$

The equations of motion are:

$$
\begin{aligned}
\dot{X}^{\mu} & =\mu X^{\mu \prime}+e P^{\mu}, & \dot{P}^{\mu} & =\left(\mu P^{\mu}\right)^{\prime}, \\
\dot{X}^{i} & =\mu X^{i^{\prime}}, & \dot{P}^{i} & =\left(\mu P^{i}\right)^{\prime} .
\end{aligned}
$$

Again, the dynamics is trivial. Taking the conformal gauge we see that the string does not move. Additionally in this case the momenta are also constant.

Let us study the Killing symmetries of this system. Considering the same generator of symmetry transformations as before (3.1), conservation of $G$ leads to:

$$
0=\dot{G}=\int \mathrm{d} \sigma\left(e P^{\mu} P^{\nu}\left(\partial_{(\mu} \xi_{\nu)}-\frac{\Lambda}{2 e} \eta_{\mu \nu}\right)+e P^{i} P^{\mu} \partial_{\mu} \xi_{i}-\gamma\left(\eta_{\mu \nu} P^{\mu} X^{\nu \prime}+P_{i} X^{i^{\prime}}\right)\right),
$$

and the Killing equations are:

$$
\partial_{\mu} \xi_{\nu}+\partial_{\nu} \xi_{\mu}=\tilde{\lambda} \eta_{\mu \nu}, \quad \partial_{\mu} \xi_{i}=0, \quad \gamma=0
$$

with conformal factor $\tilde{\lambda}=\Lambda / e=\partial_{\mu} \xi^{\mu}$. Thus we obtain the standard two dimensional conformal symmetry -as expected because the dimensionful parameter $T$ has been eliminatedwhere the "holomorphic" and "anti-holomorphic" functions have an arbitrary dependence on the transverse coordinates. On the other hand, the functions $\xi_{i}\left(X^{j}\right)$ are arbitrary.

\footnotetext{
${ }^{4}$ The tensionless limit of the relativisic string and branes has been widely dicussed in the literature, see for example [29-31].
} 


\section{Carroll $p$-brane action}

The construction of Carroll $p$-branes follows the same steps of the string case. The canonical $p$-brane action in $D$-dimensional $(D>p)$ Minkowski space is:

$$
\begin{aligned}
S_{p \text {-brane }} & =\int \mathrm{d}^{p+1} \xi\left(p \cdot \dot{x}-\tilde{s}^{\bar{a}} \mathcal{H}_{\bar{a}}-\frac{\tilde{v}}{2} \mathcal{H}\right) \\
& =\int \mathrm{d} \tau \mathrm{d}^{p} \sigma\left(p \cdot \dot{x}-\tilde{s}^{\bar{a}}\left(p \cdot \partial_{\bar{a}} x\right)-\frac{\tilde{v}}{2}\left(p^{2}+T_{p}^{2} \operatorname{det}\left(g_{\bar{a} \bar{b}}\right)\right)\right),
\end{aligned}
$$

where $\mathcal{H}=p^{2}+T_{p}^{2} \operatorname{det}\left(g_{\bar{a} \bar{b}}\right)$ and $\mathcal{H}_{\bar{a}}=p \cdot \partial_{\bar{a}} x$ are the diffeomorphism constraints and $\tilde{s}^{\bar{a}}$ and $\tilde{v}$ are the $p+1$ Lagrange multipliers. The metric $g_{\bar{a} \bar{b}}=\partial_{\bar{a}} x^{M} \partial_{\bar{b}} x^{N} \eta_{M N}(\bar{a}, \bar{b}=1, \ldots, p$ is the induced metric on the worldspace. Now we consider the Carrollian $p$-brane limit,

$$
x^{\mu}=\frac{X^{\mu}}{\omega}, \quad p_{\mu}=\omega P_{\mu} .
$$

The quantities $p \cdot \dot{x}$ and $p \cdot \partial_{\bar{a}} x$ do not change and become $P \cdot \dot{X}$ and $P \cdot \partial_{\bar{a}} X$ respectively. But the last constraint in (5.1) changes. The rescaling on the Lagrange multipliers $\tilde{s}^{\bar{a}}, \tilde{v}$ as well as the $p$-brane tension are the same as in the string case. We have: $\tilde{s}^{\bar{a}}=s^{\bar{a}}, \tilde{v}=v / \omega^{2}$ and $T_{p}=\omega T$. In the limit $\omega \rightarrow \infty$ we obtain $(i, j=p+1, \ldots, D-1)$ :

$$
\frac{\tilde{v}}{2}\left(p^{2}+T_{p}^{2} \operatorname{det}\left(g_{\bar{a} \bar{b}}\right)\right)=\frac{v}{2}\left(\eta_{\mu \nu} P^{\mu} P^{\nu}+T^{2} \gamma\right),
$$

with $\gamma=\operatorname{det}\left(\gamma_{\bar{a} \bar{b}}\right)=\operatorname{det}\left(\partial_{\bar{a}} X^{i} \partial_{\bar{b}} X^{j} \delta_{i j}\right)$. The Carroll $p$-brane action turns out to be:

$$
S_{\mathrm{C}_{p}}=\int \mathrm{d} \tau \mathrm{d}^{p} \sigma\left(P \cdot \dot{X}-s^{\bar{a}}\left(P \cdot \partial_{\bar{a}} X\right)-\frac{v}{2}\left(\eta_{\mu \nu} P^{\mu} P^{\nu}+T^{2} \gamma\right)\right) .
$$

At this point, we do not need to do a full analysis. Notice that the behaviour of the tension in the Carroll limit does not depend on $p$, the number of dimensions of the worldspace. It is the same rescaling for the point particle (in this case $T=M$ ) $[27,28]$, for the string, and so on. The substantial difference between the particle and the string is in the rescaling of $X^{1}$. If we rescale the first $p$ spatial coordinates, we can expect that the derived action will contain the same physics as that for the string. The results in the section above also hold if we add $p$ spatial extra-dimensions. This behaviour differs from the non-relativistic case, where the rescaled $p$-brane tension is $T_{p}=\omega^{1-p} T$ [3]. Notice that the behaviour of the tension in the Carroll limit does not depend on $p$, the number of dimensions of the worldspace. It is the same rescaling for the point particle (in this case $T=M)[27,28]$, for the string, and so on.

The dynamics of the Carroll $p$-brane, like the Carroll particle or the Carroll string, is also trivial. In the conformal gauge, $v=1, s^{\bar{a}}=0$, the equations of motion are

$$
\begin{aligned}
\dot{X}^{\mu} & =P^{\mu}, \quad \dot{P}^{\mu}=0, \\
\dot{X}^{i} & =0, \quad \dot{P}^{i}=\partial_{\bar{a}}\left(v T^{2} \gamma \gamma^{\bar{a} \bar{b}} \partial_{\bar{b}} X^{i}\right) .
\end{aligned}
$$




\section{Discussion and outlook}

We have constructed the action of tension-full and tensionless Carroll extended objects by doing the different Carrollian limits of a relativistic string or a $p$-brane canonical action. The action for the tension-full objects can be also constructed using the method of nonlinear realizations applied to the Carroll algebras, for the Carroll algebra see [37], for string Carroll algebras [26]. The dynamics of the ( $p$-brane) string Carroll actions are trivial independently if one considers the Carroll limit à la particle,Levy-Leblond or the (p-brane) 'stringy' Carroll limit, (5.2) (2.2). The reason for the trivial dynamics for these free Carroll dynamical objects is due to the fact that the light cone in the Carroll case collapses to the time vertical axis. In contrast, in the non-relativistic case the string and particle limit lead to different non-relativistic models, one being the vibrating string (with the string NR limit) $[1,3,4]$ and the other a non-vibrating string with a fixed lenght (particle limit) [38, 39].

If we consider the coupling of the Carroll extended objects to Carroll gauge fields as it is done for the case the particle in [27], the dynamics becomes non-trivial [40] because the interaction with the Carroll background fields opens the light cone. Extended objects with a Carroll supersymmetry that generalize the Carroll superparticle [28] can also be studied.

\section{Acknowledgments}

We acknowledge discussions with Eric Bergshoeff, Carles Batlle, Blaise Rollier, Jan Rosseel, Tonnis Ter Veldhuis. JG acknowledges Auttakit Chatrabhuti and Oleg Evin for the hospitality at the Department of Physics of Chulalongkorn University and for discussions. We acknowledge financial support from projects FPA2013-46570, 2014-SGR-104 and MDM2014-0369 of ICCUB (Unidad de Excelencia Maria de Maeztu). JG also acknowledges financial support from CUniverse research promotion project by Chulalongkorn University (grant reference CUAASC).

Open Access. This article is distributed under the terms of the Creative Commons Attribution License (CC-BY 4.0), which permits any use, distribution and reproduction in any medium, provided the original author(s) and source are credited.

\section{References}

[1] J. Gomis and H. Ooguri, Nonrelativistic closed string theory, J. Math. Phys. 42 (2001) 3127 [hep-th/0009181] [INSPIRE].

[2] U.H. Danielsson, A. Guijosa and M. Kruczenski, IIA/B, wound and wrapped, JHEP 10 (2000) 020 [hep-th/0009182] [INSPIRE].

[3] J. Gomis, K. Kamimura and P.K. Townsend, Non-relativistic superbranes, JHEP 11 (2004) 051 [hep-th/0409219] [INSPIRE].

[4] J. Gomis, J. Gomis and K. Kamimura, Non-relativistic superstrings: A New soluble sector of $A d S_{5} \times S^{5}, J H E P 12$ (2005) 024 [hep-th/0507036] [INSPIRE]. 
[5] J. Brugues, J. Gomis and K. Kamimura, Newton-Hooke algebras, non-relativistic branes and generalized pp-wave metrics, Phys. Rev. D 73 (2006) 085011 [hep-th/0603023] [INSPIRE].

[6] D.T. Son, Newton-Cartan Geometry and the Quantum Hall Effect, arXiv:1306.0638 [INSPIRE].

[7] E. Cartan, Sur les variétés à connexion affine et la théorie de la relativité généralisée. (première partie), Ann. Sci. Ecole Norm. Sup. 40 (1923) 325.

[8] P. Hořava, Quantum Gravity at a Lifshitz Point, Phys. Rev. D 79 (2009) 084008 [arXiv: 0901.3775] [INSPIRE].

[9] R. Andringa, E. Bergshoeff, S. Panda and M. de Roo, Newtonian Gravity and the Bargmann Algebra, Class. Quant. Grav. 28 (2011) 105011 [arXiv: 1011.1145] [INSPIRE].

[10] M.H. Christensen, J. Hartong, N.A. Obers and B. Rollier, Torsional Newton-Cartan Geometry and Lifshitz Holography, Phys. Rev. D 89 (2014) 061901 [arXiv:1311.4794] [INSPIRE].

[11] H.R. Afshar, E.A. Bergshoeff, A. Mehra, P. Parekh and B. Rollier, A Schrödinger approach to Newton-Cartan and Horava-Lifshitz gravities, JHEP 04 (2016) 145 [arXiv:1512.06277] [INSPIRE].

[12] T. Banks, A Critique of pure string theory: Heterodox opinions of diverse dimensions, hep-th/0306074 [INSPIRE].

[13] J. de Boer and S.N. Solodukhin, A Holographic reduction of Minkowski space-time, Nucl. Phys. B 665 (2003) 545 [hep-th/0303006] [INSPIRE].

[14] G. Arcioni and C. Dappiaggi, Exploring the holographic principle in asymptotically flat space-times via the BMS group, Nucl. Phys. B 674 (2003) 553 [hep-th/0306142] [INSPIRE].

[15] G. Barnich and C. Troessaert, Aspects of the BMS/CFT correspondence, JHEP 05 (2010) 062 [arXiv: 1001.1541] [INSPIRE].

[16] H. Bondi, M.G.J. van der Burg and A.W.K. Metzner, Gravitational waves in general relativity. VII. Waves from axisymmetric isolated systems, Proc. Roy. Soc. Lond. A 269 (1962) 21 [INSPIRE].

[17] R.K. Sachs, Gravitational waves in general relativity. VIII. Waves in asymptotically flat space-times, Proc. Roy. Soc. Lond. A 270 (1962) 103 [inSPIRE].

[18] R. Sachs, Asymptotic symmetries in gravitational theory, Phys. Rev. 128 (1962) 2851 [INSPIRE].

[19] C. Duval, G.W. Gibbons and P.A. Horvathy, Conformal Carroll groups, J. Phys. A 47 (2014) 335204 [arXiv:1403.4213] [INSPIRE].

[20] C. Duval, G.W. Gibbons and P.A. Horvathy, Conformal Carroll groups and BMS symmetry, Class. Quant. Grav. 31 (2014) 092001 [arXiv:1402.5894] [INSPIRE].

[21] J.M. Lévy-Leblond, Une nouvelle limite non-relativiste du group de Poincaré, Ann. Inst. Henri Poincaré 3 (1965) 1.

[22] V.D. Sen Gupta, On an Analogue of the Galileo Group, Nuovo Cim. 54 (1966) 512.

[23] H. Bacry and J. Levy-Leblond, Possible kinematics, J. Math. Phys. 9 (1968) 1605 [inSPIRE]. 
[24] C. Duval, G.W. Gibbons, P.A. Horvathy and P.M. Zhang, Carroll versus Newton and Galilei: two dual non-Einsteinian concepts of time, Class. Quant. Grav. 31 (2014) 085016 [arXiv: 1402.0657] [INSPIRE].

[25] M. Henneaux, Geometry of Zero Signature Space-times, Bull. Soc. Math. Belg. 31 (1979) 47 [INSPIRE].

[26] J. Gomis and F. Passerini, unpublished notes.

[27] E. Bergshoeff, J. Gomis and G. Longhi, Dynamics of Carroll Particles, Class. Quant. Grav. 31 (2014) 205009 [arXiv:1405.2264] [INSPIRE].

[28] E. Bergshoeff, J. Gomis and L. Parra, The Symmetries of the Carroll Superparticle, J. Phys. A 49 (2016) 185402 [arXiv: 1503.06083] [InSPIRE].

[29] J. Gamboa, C. Ramirez and M. Ruiz-Altaba, Field Theory of Null Strings and $P^{-}$branes, Phys. Lett. B 231 (1989) 57 [InSPIRE].

[30] I.A. Bandos and A.A. Zheltukhin, Null super p-branes quantum theory in four-dimensional space-time, Fortsch. Phys. 41 (1993) 619 [INSPIRE].

[31] A.A. Zheltukhin, A Hamiltonian of null strings: An invariant action of null (super)membranes, Sov. J. Nucl. Phys. 48 (1988) 375 [INSPIRE].

[32] D.M. Hofman and B. Rollier, Warped Conformal Field Theory as Lower Spin Gravity, Nucl. Phys. B 897 (2015) 1 [arXiv: 1411.0672] [INSPIRE].

[33] J. Hartong, Gauging the Carroll Algebra and Ultra-Relativistic Gravity, JHEP 08 (2015) 069 [arXiv: 1505.05011] [INSPIRE].

[34] J. Gomis and F. Passerini, Rotating solutions of non-relativistic string theory, Phys. Lett. B 617 (2005) 182 [hep-th/0411195] [INSPIRE].

[35] S.R. Coleman, J. Wess and B. Zumino, Structure of phenomenological Lagrangians. I, Phys. Rev. 177 (1969) 2239 [INSPIRE].

[36] C.G. Callan Jr., S.R. Coleman, J. Wess and B. Zumino, Structure of phenomenological Lagrangians. II, Phys. Rev. 177 (1969) 2247 [INSPIRE].

[37] T.E. Clark and T. ter Veldhuis, AdS-Carroll Branes, arXiv: 1605.05484 [INSPIRE].

[38] C.F. Yastremiz, Galilean extended objects, Class. Quant. Grav. 9 (1992) 2395 [InSPIRE].

[39] C. Batlle, J. Gomis and D. Not, Galilean Symmetries of the non-relativistic limits of a relativistic string, work in progress.

[40] E.Bergshoeff, J. Gomis, B. Rollier, J. Rosseel and T. ter Veldhuis, Carroll versus Galilei Gravity, work in progress. 